\title{
Epistemología en Enfermería. Una revisión del acto de cuidar desde Michel Foucault, el modelo de Roper, Logan \& Tierney y el concepto de "bildung" de Gadamer
}

\author{
Rosana Elisabet Firpo *
}

\section{Resumen}

Desde mediados del siglo pasado el sustento teórico de la disciplina Enfermería se nutrió a través de la elaboración de teorías y modelos de diferente rango y orientación, la mayoría de ellos originados en los Estados Unidos (Henderson, Peplau, Rogers, Leininger, Orem, por citar sólo los más conocidos). De hecho, en la Universidad de lowa se desarrolló una taxonomía diagnóstica con el propósito de homogeneizar el lenguaje enfermero ${ }^{1}$ que al día de hoy cuenta con una importante aceptación a nivel mundial en lo que respecta al desarrollo de los planes de cuidado.

Sin embargo, el intento de imponer esos modelos teóricos en el contexto de América Latina -más que nada por parte de los ámbitos académicos- ha sido muy resistido. A fin de revisar el sustrato filosófico de la adopción de un modelo teórico para la disciplina Enfermería, desde este trabajo se parte de la concepción de persona desde Michel Foucault como ser de vida, trabajo y lenguaje y se recurre al concepto de bildung (Gadamer, 2004) entendido como "edificación" para orientar una necesaria revisión en lo que hace al objeto de estudio y trabajo de la Enfermería.

A partir de hacer énfasis en la persona (familia/comunidad por extensión) destinataria de los cuidados, se propone explicitar la articulación de los tres planos del cuidado a fin de dotar identidad a la disciplina Enfermería al superar el enfoque instrumental y tecnocrático que en general ha teñido a la profesión.

En este camino, se acuerda con Richard Rorty (1979) en la necesidad de mantener el diálogo -en este sentido la taxonomía diagnóstica de la NANDA sería un obstáculo- y se postula un objeto de estudio interdisciplinar que ponga a conversar a las diferentes disciplinas involucradas en el acto de cuidar y se considera que desde el Modelo de Roper, Logan y Tierney, al incluir los determinantes sociales de la salud, es posible tender puentes no sólo hacia la interdisciplina sino además hacia la intersectorialidad.

Palabras clave: $\quad$ Epistemología - Cuidado - Enfermero - Modelo de Roper, Logan y Tierney - Bildung

\footnotetext{
${ }^{1}$ NANDA (North American Nursing Diagnosis Association)
} 


\title{
Epistemology in Nursing. A review of the act of caring from Michel Foucault, the model of Roper, Logan \& Tierney and the concept of "bildung" of Gadamer
}

\author{
Rosana Elisabet Firpo *
}

Abstract

Since the middle of the last century, the theoretical support of the Nursing discipline was nurtured through the development of theories and models of different rank and orientation, most of them originated in the United States (Henderson, Peplau, Rogers, Leininger, Orem, by cite only the best known). In fact, at the University of lowa a diagnostic taxonomy was developed with the purpose of homogenizing the nursing language (NANDA (North American Nursing Diagnosis Association) that today has an important worldwide acceptance with regard to the development of care plans.

However, the attempt to impose these theoretical models in the context of Latin America - more than anything else on the part of academia - has been very resisted. In order to review the philosophical substratum of the adoption of a theoretical model for the Nursing discipline, this work starts from Michel Foucault's conception of the person as a being of life, work and language and uses the concept of bildung (Gadamer, 2004) understood as "edification" to guide a necessary revision regarding the object of study and work of Nursing.

Starting from emphasizing the person (family / community by extension) receiving care, it is proposed to make explicit the articulation of the three planes of care in order to give identity to the Nursing discipline by overcoming the instrumental and technocratic approach that in general it has colored the profession.

Along this path, he agrees with Richard Rorty (1979) on the need to maintain dialogue in this sense, the NANDA diagnostic taxonomy would be an obstacle - and an interdisciplinary object of study is postulated that puts the different disciplines involved in conversation in the act of caring and it is considered that from the Roper, Logan and Tierney Model, by including the social determinants of health, it is possible to build bridges not only towards interdiscipline but also towards intersectorality.

Keywords: $\quad$ Epistemology - Care - Nurse - Roper, Logan and Tierney model Bildung 


\section{Introducción}

Desde este trabajo se asume que, de los cuatro términos que componen el metaparadigma en Enfermería es el de persona el que tracciona a los restantes (salud, enfermería y entorno) en pos de la definición del objeto de trabajo y estudio de la disciplina más allá de que además se señale que "aunque el metaparadigma es ampliamente aceptado, la inclusión de Enfermería como un concepto importante de la disciplina es tautológica" (Smith y Liehr, 2018: 4)

La Enfermería tiene identificado desde hace ya bastante tiempo al cuidado como el objeto de trabajo y estudio de la disciplina. Por ejemplo se puede citar a Keroauc y colaboradores quienes aseveran que el centro de interés de la disciplina es "el cuidado de la persona que, en interacción continua con su entorno, vive experiencias de salud" (1996:62) o Bruce, Rietzel y Lim que afirman que "la primacía de cuidar se considera el valor central en el corazón del mandato social que los Enfermeros tienen" (2014:69) entre otros/as autores ${ }^{2}$.

De todas formas, quizá llegó el momento de preguntarse si el cuidado es el objeto que se corresponde con el avance del entendimiento acerca del proceso saludenfermedad en el siglo XXI y en América Latina. Acaso sea la hora de revisar los supuestos que subyacen en la necesidad de identificar un objeto propio que permitiera diferenciar el aporte de la Enfermería en el equipo de salud. Tal vez esa ya sea una etapa superada y los tiempos actuales están requiriendo una mirada más comprehensiva de los determinantes de la salud de las personas y las comunidades sobre todo cuando se admite la mutación del, hasta hace poco tiempo, denominado proceso salud/enfermedad/atención al proceso salud/enfermedad/cuidado ${ }^{3}$.

Se sostiene que la relación entre la filosofía y los cuidados enfermeros "se da principalmente a partir de que éstos se enfocan en cuestiones que hacen a la trascendencia y la singularidad de la vida de las personas que se ven afectadas por temas como la muerte, el dolor, la enfermedad" (Enrici A y col, 2015: 129). En función de lo expuesto anteriormente se propone partir del concepto de persona desde Michel Foucault y articular al que sería el plano filosófico del acto de cuidar, un plano teórico desde el Modelo de Roper, Logan y Tierney y, de esta forma, superar el plano meramente técnico que corresponde a la realización de técnicas y procedimientos derivados en general de la indicación médica y de la atención a una patología.

Esta propuesta se solapa con la estrategia planteada por Smith y Liehr para la "enseñanza de la epistemología en Enfermería con un enfoque reflexivo y de aprendizaje

\footnotetext{
${ }^{2}$ También Durán de Villalobos (2002) menciona como definición de la disciplina Enfermería "el estudio del cuidado de la experiencia o vivencia de la salud humana"

${ }^{3}$ Michalewicz A, Pierri C y Ardilla-Gómez S (2014)
} 
significativo (que) fue estructurada para su aplicación con estudiantes de posgrado" (Gómez O, Carillo G y Cárdenas C, 2017: 437) ${ }^{4}$

\title{
La articulación de los planos técnico, teórico y filosófico en el acto de cuidar
}

\author{
“Nacer, crecer, morir, enfermar: cosas tan simples y tan constantes en apariencia. Los hombres, \\ empero, han desarrollado en relación con ellas actitudes complejas y cambiantes que no sólo modifican el \\ sentido que se les da sino también, a veces, las consecuencias que acarrean" \\ Michel Foucault, "La inquietud por la verdad", p 228
}

a. El plano filosófico:

El artículo de Bruce, Rietzel y Lim (2014) expone muy claramente la relación entre Filosofía y Enfermería; los autores por una parte advierten que "en un contexto sociopolítico donde los valores de la productividad, la eficiencia y la mercantilización del conocimiento y del cuidado están en aumento, no es de extrañar que la búsqueda de conocimiento filosófico en Enfermería esté amenazado" (2014: 66). También distinguen entre la filosofía analítica y la continental; dentro de esta última se incluye a los dos autores que se toman de referencia en este artículo: Foucault y Gadamer.

Cuando considera a la persona como ser de vida, trabajo y lenguaje, Foucault (2014) remite a tres ciencias: la Biología, la Economía y la Lingüística. En otro texto (Hermenéutica del sujeto, 1996) el filósofo alude a la dietética, la economía y la erótica como las tres grandes líneas de evolución de la noción del cuidado de sí mismo.

Es éste el marco filosófico propicio para articular un modelo teórico de la disciplina Enfermería. Cuando Smith y Liehr proponen la escalera de abstracción, el peldaño filosófico se correspondería con la visión del mundo según lo describen en su artículo Gómez, Carrillo y Cárdenas (2017: 440).

Cabe recordar que según Bruce, Rietzel y Lim "la filosofía continental (...) está interesada en entender la experiencia humana (y) aumenta la capacidad de los Enfermeros para teorizar y esto proporciona una manera de pensar acerca de su práctica" (2014: 67) lo que deriva en el concepto de cuidado holístico en el ejercicio de la profesión al pensar a la persona más allá de su patología o las rutinas del turno.

\footnotetext{
${ }^{4}$ Cabe resaltar que Smith y Liehr no están traducidas al castellano. El artículo de referencia es del año 2017 y lo planteado en este trabajo ya se presentó en un Congreso Nacional en el mes de octubre del año 2016 y cuenta con al menos dos años de trabajo previo. El conocimiento del artículo de Gómez, Carrillo y Cárdenas se produce en una mesa de examen en diciembre del año 2017 cuando la estudiante Carolina Delgado lo incluye en la bibliografía de su presentación. De todas formas, la estrategia de Smith y Liehr se orienta en un sentido diferente al aquí propuesto y no se mencionan autores ni desde el plano filosófico ni teórico.
} 


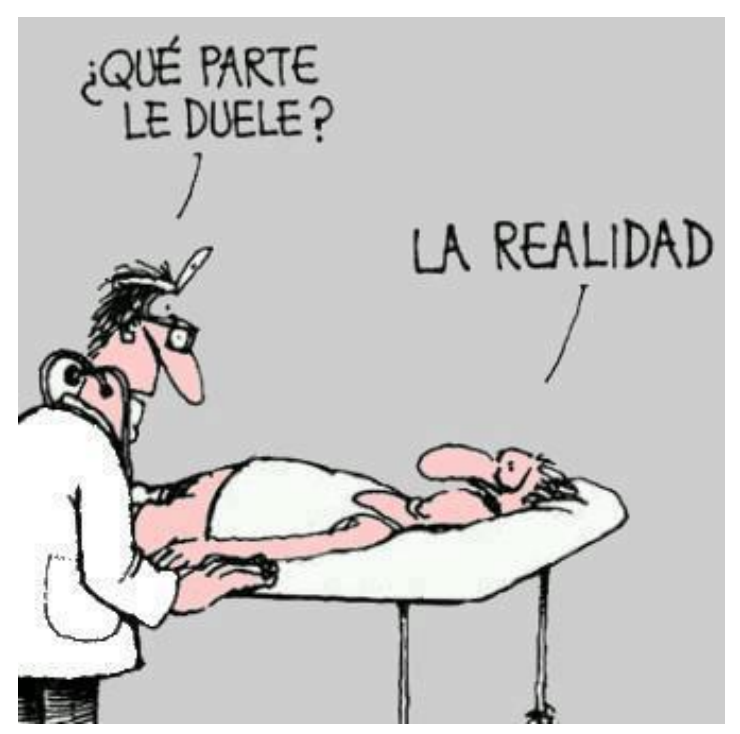

A través del "chiste" se intenta una aproximación a darnos cuenta de la necesidad de personalizar el cuidado enfermero y la elaboración de planes de cuidado que al decir de Ballantyne "sean específicos del contexto y centrados en el paciente" (2016: 51) Esta autora también advierte -en consonancia con Debout (2008)- que los planes de atención tienen un componente económico al mencionar la relación costoefectividad y la característica de rentabilidad de los mismos ${ }^{5}$ https://i.pinimg.com/originals/36/7c/5a/367c5ad6ffe87b25c4ee8b0ef08cba0c.jpg

b. El plano teórico.

Desde este trabajo se elige el Modelo de Vida de Roper, Logan y Tierney para el desarrollo del plano teórico por su versatilidad para ser aplicado en los diferentes ámbitos del ejercicio profesional y porque desde sus componentes se articula al plano filosófico planteado. El Modelo aparece entre las "Teorías de importancia histórica" en el libro de Martha Alligood quien afirma que el desarrollo de conocimiento en Enfermería "(...) fue un objetivo principal propuesto por los líderes de la profesión en el siglo XX porque las enfermeras buscaban mejorar la práctica y obtener el reconocimiento de la Enfermería como profesión" (2011: 3)

Según Ballantyne "los modelos ofrecen una variedad de creencias y valores para guiar a los Enfermeros a través de resolución de problemas y proporcionar orientación sobre lo que es relevante e importante" (2016: 52). Específicamente el Modelo de Vida tiene cinco componentes: la etapa de la vida, doce actividades vitales, factores que influyen en las actividades vitales (desde los cuales se incorporan los determinantes sociales de la salud), el nivel de dependencia-independencia en cada una de las actividades vitales y la individualidad de la vida.

Concretamente la articulación con el plano filosófico sería la siguiente: La persona como ser de vida, nace y muere, es decir, transita un ciclo vital (contando desde la concepción hasta la muerte). En ese ciclo vital se suceden diferentes etapas de la vida en las cuales la persona atraviesa crisis de desarrollo como ser la adolescencia o la menopausia o vive experiencias de salud tales como un embarazo, una enfermedad, un accidente; al atravesar esas experiencias las personas tienen diferentes formas de 
afrontamiento ya sea por la cultura en la que viven, por hechos previos relacionados, por la red de acompañamiento familiar y social de la que dispongan, entre otras cosas.

Como ser de trabajo, se admite que habitualmente las personas tienen una ocupación y un entretenimiento; ambas situaciones (que además van a ir variando según las etapas de la vida) pueden influir en su estado de salud-enfermedad-cuidado; no es lo mismo trabajar de minero que de docente universitario y no es igual tener por entretenimiento armar rompecabezas que practicar deportes de riesgo. En este sentido se puede pensar en la cobertura social, en leyes de protección a las personas, en su identidad en tanto su inserción en el sistema de producción por dar algunos ejemplos.

Por último, como ser de lenguaje, podría aceptarse que la persona ama y lucha; el ser humano tiene pasiones que lo llevan a considerar hábitos de vida o tomar decisiones que también influyen en su bienestar y en esto más que nada se materializa (si puede decirse así) la individualidad de la vida.

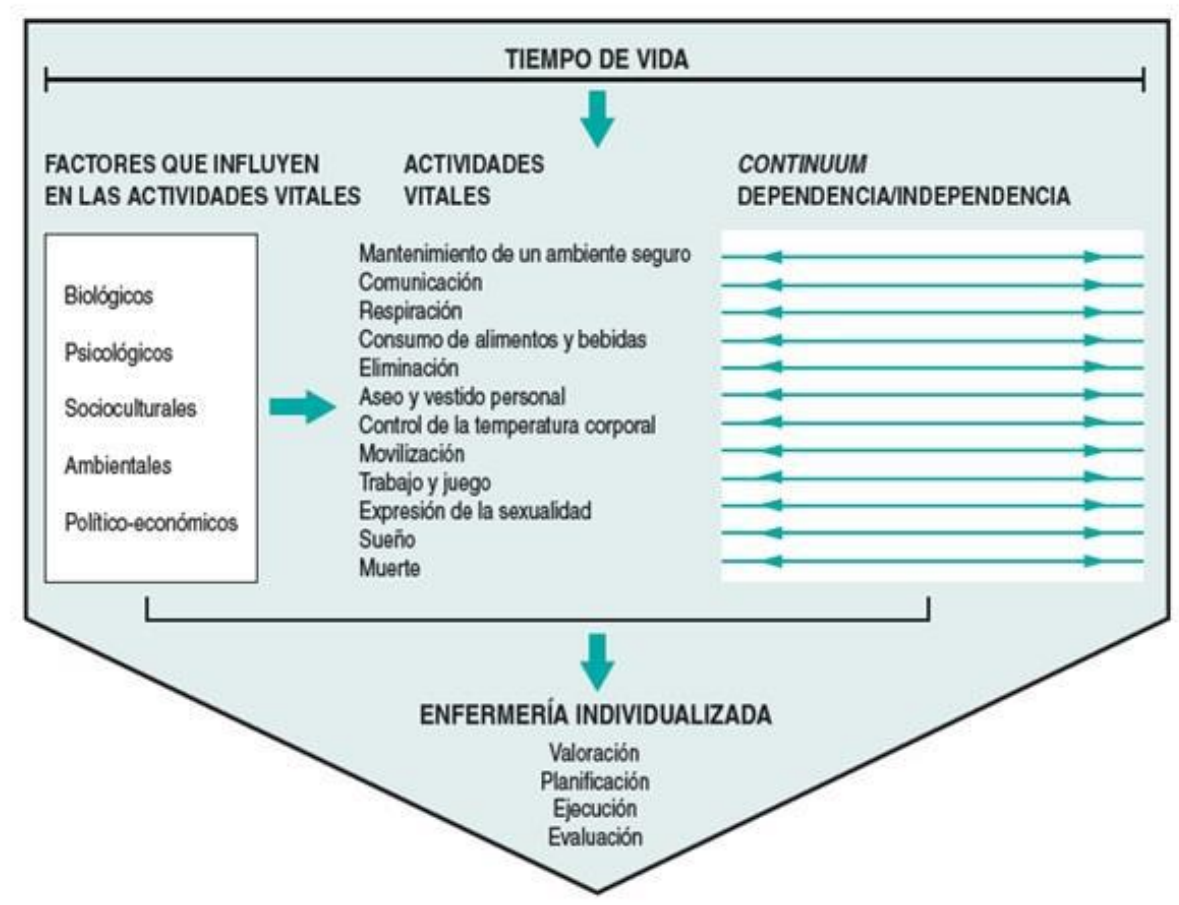

Cabe resaltar que el hecho de contar con modelos teóricos disciplinares es un aporte que Enfermería puede hacer a otros espacios como lo destaca Stonehouse: "Ios trabajadores de apoyo (Partería) trabajan en estrecha colaboración con colegas de Enfermería que implementan el proceso de Enfermería para brindar atención de calidad" (2017: 1)

c. Plano técnico:

En este sentido, Ballantyne (2016) reflexiona sobre las formas de trabajo en Enfermería y señala que el hecho que estén en base a las rutinas de los servicios refleja un sistema basado en las tareas y por lo tanto el cuidado es procedimiento centrado.

Por su parte, Bruce, Rietzel y Lim -que citan a Sulavikova- advierten sobre el "acercamiento instrumental de los programas de estudio (...)" en contraste al desarrollo 
del pensamiento crítico/reflexivo. En la actualidad, el diseño de escenarios para la estrategia educativa de la Simulación Clínica intenta promover el aprendizaje significativo.

También Medina Moya y do Prado acuerdan (y critican) que "la práctica de la enseñanza en Enfermería es marcadamente instrumental y tecnológica" (2015: 619). El hecho de privilegiar este plano en la docencia y en el ejercicio profesional tiende a reforzar la racionalidad instrumental a expensas del aprendizaje reflexivo a través del cual se intenta abordar no sólo las competencias procedimentales sino también las cognitivas (a través del plano teórico) y las relacionales (plano filosófico).

Es por esto que se insiste en la importancia del concepto de persona sobre los demás; pasar de un sistema procedimiento centrado a uno centrado en la persona. Según Bruce, Rietze y Lim es el pensamiento filosófico el que contribuye a este cambio de paradigma: "Las conceptualizaciones sobre la naturaleza de los buenos cuidados de enfermería y el conocimiento enfermero pueden ser útiles para que Enfermería disminuya la práctica reduccionista y mecanicista (...)" (2014: 69). De esta forma se cuestiona la atención fragmentada en base a los procedimientos y se orienta hacia un cuidado integral que promueva el crecimiento profesional desde las diferentes funciones de Enfermería: no sólo la asistencial sino integrar la docencia, la investigación y la gestión.

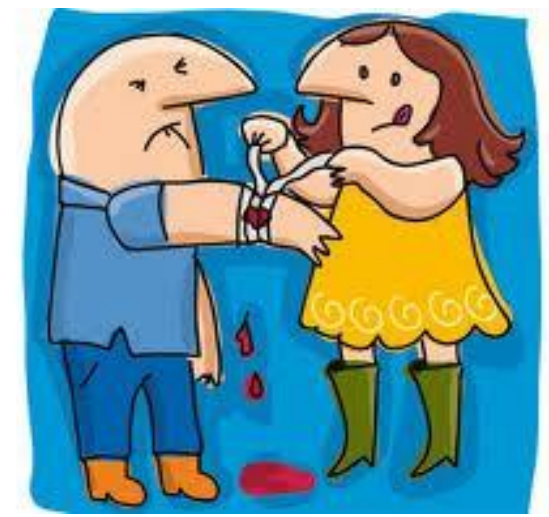

Esta imagen remite a la apreciación de José Medina: “actualmente, en la práctica de Enfermería se observa que el cuidado es identificado en el hacer monótono, lineal y sin sentido, dentro de la racionalidad técnica y el enfoque biomédico" (citado en Báez-Hernández, 2009:129)

Un ejemplo concreto sería el siguiente:

- Plano técnico: hay que realizar una curación (competencia procedimental)

- Plano teórico: desde el Modelo de Roper, Logan y Tierney se identifica la actividad vital de Mantenimiento del Entorno Seguro, se formula un diagnóstico de Enfermería (Riesgo de infección relacionado con solución de continuidad de la piel), se establece un objetivo (la persona será capaz de disminuir el riesgo de infección) y se enumeran actividades de Enfermería (valorar las características de la herida, administrar la medicación, indagar acerca del dolor, realizar el procedimiento adecuado, registrar). Se valoran las competencias cognitivas.

- Plano filosófico: ¿quién es la persona que tiene la herida y necesita una curación? ¿Un niño de 2 años por maltrato familiar? ¿Una puérpera que tuvo un embarazo normal y se 
decidió hacer cesárea por falta de progresión del parto? - (herida limpia en entorno quirúrgico)- ¿un joven que trabaja en situación de informalidad, tuvo un accidente laboral y resultó con una amputación (herida sucia)? ¿Una persona mayor que tiene una úlcera por déficit de autocuidado de Diabetes? Desde este plano se tiende al desarrollo de las competencias relacionales.

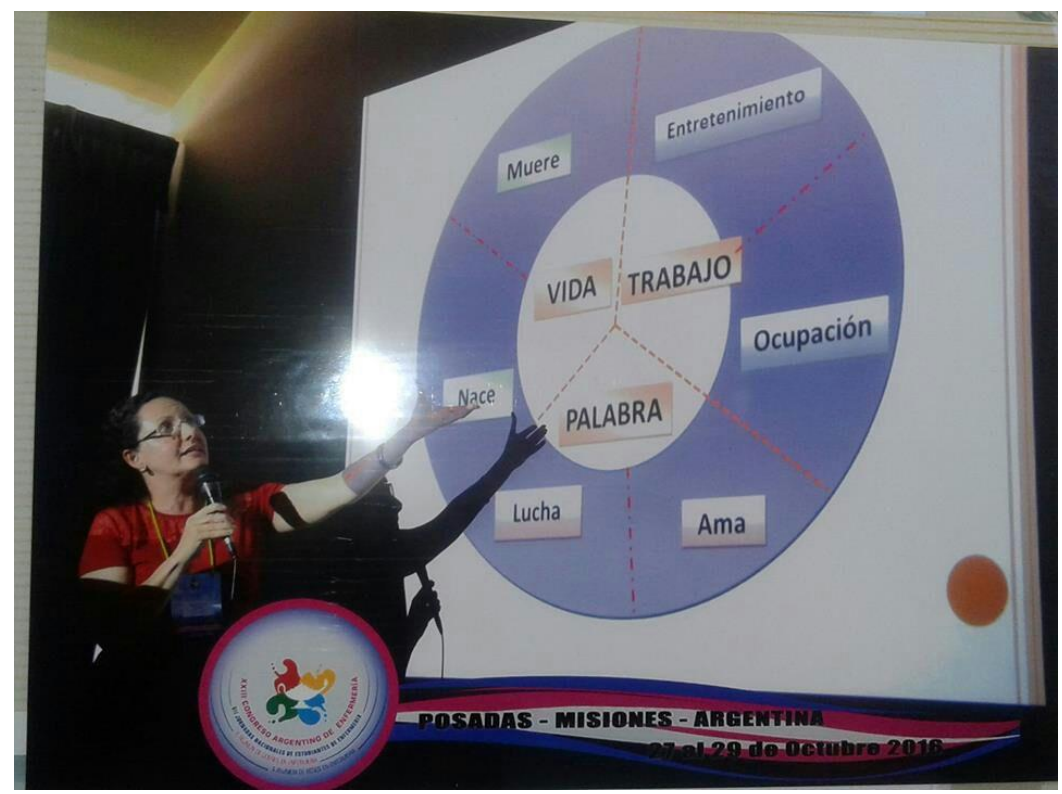

La imagen corresponde al Congreso Argentino de Enfermería realizado en el año 2016. Las líneas punteadas indican que "existe un continuo transvase" entre cada dominio tal como lo menciona Foucault (1982: 50) para los tres ámbitos de la práctica de uno mismo: el cuerpo, el entorno y la casa.

\section{El cuidado enfermero. En búsqueda de una identidad latinoamericana en el siglo} XXI

“Nada se habrá comprendido de la enfermedad en tanto que no se reconozca su extraña semejanza con la guerra y el amor, sus compromisos, sus fintas, sus exigencias, esa amalgama tan extraña como única producida por la mezcla de un temperamento y un mal" Marguerite Yourcenar, "Memorias de Adriano", p 235

La pregunta puede parecer inverosímil ¿y si no es el cuidado? ¿y si todos estos años sólo hemos repetido un discurso generado en contextos muy diferentes de los que nos toca actuar cada día? ¿Qué tan factible resulta imaginar un objeto de estudio y trabajo para Enfermería más congruente con la realidad cotidiana en América Latina? Un objeto no sólo disciplinar sino interdisciplinar.

Desde la Epidemiología se indica el camino que bien vale para la Enfermería: la necesidad de incorporar un paradigma que conduzca a la transformación de la realidad. Afirma Breilh, que, en la actualidad, el mayor desafío es "perfeccionar nuestra conciencia objetiva sobre los nuevos problemas de una realidad muy compleja y caracterizada por 
una espiral de creciente inequidad, pero hacerlo simultáneamente por una conciencia sobre la subjetividad como herramienta de impulso colectivo" (2009:30).

Esta cuestión de la inequidad no pasa desapercibida cuando Bruce, Rietzel y Lim apuestan a incorporar la filosofía al pensamiento enfermero ya que de esta forma los/as profesionales contarían con herramientas para "garantizar el acceso equitativo a los servicios de salud para todas las personas, abogando por la equidad y (...) la atención por valores personales y profesionales de la justicia social" (2014: 69)

Se podría decir que Enfermería en la actualidad se encuentra en una tensión entre delimitar su área de acción y ser partícipe de un equipo interdisciplinario con identidad profesional propia. En su artículo, Christophe Debout afirma que "una disciplina requiere que sus especialistas sean capaces de crear su zona de autonomía" (2008: 72). Y como se expresó anteriormente, la autonomía se daría al superar el plano técnico y articular los planos teórico y filosófico.

En la medida que Enfermería pueda delimitar su área de acción será capaz de contribuir al campo de la salud en el sentido en que lo plantean Michalewicz, Pierri y Ardilla-Gómez: "no sólo ni principalmente como un escenario de prácticas, sino como un terreno de luchas simbólicas acerca de las formas de aproximarse al modo en que las personas nacen, enferman, padecen y mueren y respecto a las respuestas sociales frente a tales procesos" (2014: 218, citan a Stolkiner y Ardilla-Gómez, 2012)

En relación a lo anterior, la pregunta que orienta este trabajo es la siguiente: ¿cómo se construye desde la docencia y en la práctica cotidiana -ya sea comunitaria u hospitalaria- el sujeto destinatario de los cuidados de Enfermería? En realidad, esta pregunta contiene en sí misma varias aristas.

En principio cabe aclarar que el término más apropiado no sería construcción o constitución sino el de bildung, traducido como edificación, al que Gadamer atribuye siguiendo a Hegel- la característica de "mantenerse abierto a lo que es el otro, a otros puntos de vista más universales" (2004: 15)

En este sentido, la incorporación del plano filosófico implica no solo pensar al otro como sujeto de cuidado sino también pensarse a sí mismo como profesional. Es por esto que Ballantyne (2016) resalta que a través de los planes de cuidado se benefician tanto los pacientes como Enfermería. Cabe resaltar que el diseño de un plan de cuidados requiere de un modelo teórico en el cual posicionarse y pensar desde una visión integral, filosófica, el acto de cuidar.

$\mathrm{Si}$, como profesional, la función cotidiana consiste en la sumatoria de procedimientos derivados de las indicaciones médicas o que responden a las rutinas del servicio (control de signos vitales, administración de medicación, realización de higiene), dentro de la racionalidad instrumental, eso impacta de forma negativa en la subjetivación y obstaculiza el ejercicio de los roles ya que se limita sólo al de proveedor/a de cuidados directos en detrimento de otros como el de enlace, docente, comunicador/a, defensor/a de los derechos de las personas, consejero/a, líder, investigador/a. 
En cuanto al sujeto destinatario de los cuidados cabe preguntarse sobre si es disciplinar o necesariamente interdisciplinar en acuerdo con Jaime Breilh en tanto "relación entre saberes académicos que comparten un espacio" y particularmente con el tipo de relación definido como Multidisciplinaridad: "integración de disciplinas a través del análisis interdisciplinario en un proceso científico solidario para la construcción de un proyecto en común" (2009:294) ${ }^{6}$.

En lo que hace a este juego por una parte de definir lo propio de la disciplina enfermería y por otra parte de participar de la necesaria interdisciplina en la atención de la salud, Debout cita a Ben David quien sugiere que "la disciplina trabajará para identificar y explorar sus fenómenos de interés (y) tenderá gradualmente a señalar a la disciplina emergente y distinguirla de sus vecinas" (2008: 73).

Otra de las aristas a las que remite la pregunta sobre el sujeto destinatario de los cuidados da cuenta del concepto de alteridad. Como afirma Debout (2008:76) "como cualquier ciencia aplicada que tiene por objeto dispensar cuidado a otro ser humano, los cuidados enfermeros no pueden concebirse sin la relación que une al beneficiario de los cuidados y el profesional que los brinda"

En consecuencia, puede afirmarse que existe un camino en común entre el aporte de la Filosofía en Enfermería y la noción de bildung: el imperativo relacional de la Enfermería atribuido por Bruce, Rietze y Lim (2014: 69) se relaciona estrechamente con la conceptualización de Richard Rorty acerca de que "la filosofía edificante aspira a mantener una conversación más que a descubrir la verdad" (1979: 337).

Por lo expuesto en el párrafo anterior, no resulta arriesgada la afirmación de que cuando se aplica la taxonomía de la NANDA elaborada en base a los patrones de Marjorie Gordon se está, no sólo clausurando el diálogo necesario para edificar una identidad profesional, disciplinar -es decir se está yendo exactamente en el sentido opuesto ya que "el resultado de bildung (...) surge de un proceso interno de formación y cultivo" (Gadamer: 9), sino que además se está afectando la alteridad.

Se hizo mención al comienzo acerca de la popularidad que tiene la taxonomía de la NANDA que se adopta a fin de proponer un lenguaje enfermero. Desde este trabajo se acuerda más bien con la percepción de Ballantyne para quien "quizás estamos tratando de estandarizar aspectos de la atención de Enfermería que no pueden ser estandarizados" (2016: 56)

En ese trayecto entre la necesidad de delimitar el campo de acción y contribuir con identidad propia al campo de la salud, es muy probable que también se recurra al concepto de bildung en el sentido de que se "requiere sacrificar la particularidad por el bien de lo universal" (Gadamer: 11). Podría hablarse de que la disciplina enfermería ya no

\footnotetext{
${ }^{6}$ Breilh también enumera: pluridisciplinaridad, multidisciplinaridad, epidisciplinaridad, metadisciplinario y trandisciplinario. Si bien también pueden aplicarse a este caso los tipos de relación de interdisciplina y transdisciplina, se eligió el de multidisciplina por la incorporación del concepto de solidaridad que se considera imprescindible en las circunstancias actuales de precariedad en las condiciones de vida y de trabajo.
} 
se ciñe al cuidado enfermero como terreno propio sino que resigna su característica definitoria en pos de cooperar en un proyecto más ecuménico: la "asistencia sociosanitaria para contribuir al bienestar de las personas, familia y comunidad"

Asistencia proviene de assistere que significa "estar cerca"7 , vocablo que remite por un lado a una matriz necesariamente dialógica y por otro al término ayuda, tan recurrente cuando se definen las funciones de la Enfermería; sociosanitaria por el reconocimiento que las condiciones sociales impactan en el bienestar de las personas ${ }^{8}$ y el bienestar viene dado como concepto subjetivo definido a partir de los sujetos destinatario de los cuidados.

Smith y Liehr son muy concretas respecto a esto y reconocen que "algunos sugieren que ya no es necesario diferenciar el conocimiento y establecer límites de disciplina porque los equipos interdisciplinarios realizarán investigaciones sobre problemas comunes eliminando la necesidad de establecer límites de disciplina" (2018: 11) y además cuando refieren a que las teorías de rango medio pueden ayudar a las diferentes disciplinas a trabajar en fenómenos de interés compartido claramente se puede asociar a esto el concepto de cuidado.

Sin embargo, hay un problema a sortear. Michalewicz, Pierri y Ardilla-Gómez identificaron cinco categorías emergentes en la bibliografía en derredor del término cuidado (polisemia) aunque al momento de privilegiar el hecho de establecer un vínculo resaltan dos de los usos: el que apunta a la integralidad en la atención y el que propone la reorganización de los servicios (2014: 223). Los autores también recurren a Tejada de Rivero en tanto diferenciar entre los conceptos de cuidado y atención en lo que hace al tipo de relación que se establece; para el cuidado se identifican relaciones horizontales, simétricas, participativas e intersectoriales.

Podría afirmarse que la disciplina Enfermería conlleva una ventaja al contar con modelos teóricos y de esta forma adherir a lo que resalta Ballantyne desde el Nursing and Midwifery Council (NMC, el Consejo de la Enfermería y la Partería) en relación a que los Enfermeros deben mantener informados a sus colegas -cosa que comparte con el texto de Stonehouse-.

Específicamente de la etapa de valoración del Proceso de Atención de Enfermería se puede orientar información para diferentes tipos de diagnósticos e intervenciones para los demás trabajadores/as no sólo del sector salud sino también por ejemplo del campo de Trabajo Social o Psicopedagogía en la escuela o del área de la justicia si se necesita por ejemplo tramitar un expediente o trámite que permita mejorar la calidad de vida. El aporte de la filosofía viene dado porque "aumenta a capacidad de los enfermeros para

\footnotetext{
${ }^{7}$ Báez-Hernández y col (2009:132) citan a Aguilar HR: “el profesional de enfermería requiere de sensibilidad, intelecto, disciplina y conocimientos sistematizados para identificar $y$ atender las necesidades $y$ posibilidades humanas, mediante un contacto personal cercano con el paciente"

${ }^{8}$ Recordar el exquisito poema de Bertolt Brecht "Palabras de un obrero a un médico" que comienza con un categórico "Nosotros sabemos lo que nos enferma" y luego discurre entre las condiciones de vida como el trabajo en exceso, la vivienda, la alimentación.
} 
teorizar, proporciona una manera de pensar acerca de su práctica, una manera de dar sentido, articular, criticar la práctica de enfermería" (Bruce, Rietze y Lim, 2014:68).

En la actualidad, el desarrollo de la estrategia educativa de la Simulación Clínica está brindando una enorme oportunidad al interpelar las prácticas pedagógicas y por ende revisar la relación docencia-servicio y dotar a los/as futuros/as profesionales de competencias para integrar un equipo interdisciplinario con un rol diferenciado desde lo cualitativo.

\section{A modo de conclusión}

Smith y Liehr (2018) señalan el riesgo de permanecer al margen de las disciplinas profesionales $y$, por lo tanto, el peligro de ser consumida o ignorada si no presta suficiente atención a la singularidad del campo de investigación y práctica; es decir si no se trabaja en delimitar el objeto de trabajo y estudio de tal forma que se pueda visibilizar la contribución específica en el equipo de salud.

Como se mencionó anteriormente, el contexto sociocultural en el que se produce el lenguaje enfermero estandarizado es muy ajeno a la realidad en América Latina y, por otra parte, la taxonomía propuesta por la NANDA respondería más a la presión de grupos económicos sobre el sistema de salud ${ }^{9}$ que al propósito de fomentar el desarrollo profesional.

El modelo propuesto por las teóricas de la Universidad de Edimburgo resulta más versátil, adecuado a los diferentes ámbitos de actuación de los/as profesionales de la Enfermería y sobre todo orienta a integrar equipos de trabajo. Un ejemplo lo brinda el artículo de Stonehouse (2017) donde detalla el Modelo de Roper, Logan y Tierney para trabajadores de la Partería y destaca que "los trabajadores de apoyo trabajan en estrecha colaboración con colegas de Enfermería que implementan el proceso de Enfermería para brindar atención de calidad" (p. 1).

El peso específico y la visibilidad de la disciplina Enfermería en los equipos de salud dependerá, en gran parte, de la forma en que sustenten desde lo teórico y lo filosófico la prestación del cuidado. En la actualidad, el reclamo por ser considerados/as profesionales se juega en ese sentido, en el sentido de pensar el cuidado desde un colectivo que ponga en el centro a las personas/familias/comunidades y establezca relaciones horizontales en el campo de la salud

\footnotetext{
${ }^{9}$ Debout C (2008) Se alude a la integración de la actividad de Enfermería en los sistemas de información médicos. Si la atención a la salud se reduce a la asistencia médica el sistema está orientado a recuperar los gastos producidos por los tratamientos y los métodos diagnósticos empleados; en ese caso, los cuidados enfermeros se restringen a las técnicas, procedimientos y rutinas del servicio que se privilegian por sobre el acto de cuidar.
} 


\section{Referencias bibliográficas}

ALLIGOOD, M. y MARRIMER TOMEY, A. (2011). Modelos y Teorías en Enfermería. 7ma edición. España: ELSEVIER.

BÁEZ-HERNÁNDEZ, F., NAVA-NAVARRO, V., RAMOS-CEDEÑO, L., MEDINA-LÓPEZ, O. (2009). "El significado de cuidado en la práctica profesional de Enfermería". En Chía, Año 9, Vol 9 (2): 127-134 disponible en www.scielo.org.co. Los autores citan un artículo de Medina J del año 1999.

BALLANTYNE, H. (2015). "Developing nursing care plans" en: Nursing Standard, Vol 30, N 26: 51-56 disponible en www.researchgate.net

BREILH, J. (2009). Epidemiología Crítica. Ciencia emancipadora e interculturalidad. Argentina: Lugar Editorial.

BRUCE, A., RIETZE, L., LIM, Á. (2014). “Undertanding Philosophy in a Nurse's World: What, Where and Why?" en: Nursing and Health 2 (3): 65-71. Disponible en http://www.hrpub.org

DEBOUT, C. (2008). "Sciences des soins infirmiers: réflexions épistemologiques sur le projet d'une discipline" en: Recherche en soins infirmiers, ( $N$ 93), p 72-82. DOI 10.3917/rsi.093.0072, disponible en www.cairn.info

ENRICl, A., CISELLI, G., FIRPO, R., TRIVIÑO, M. (2015). Arte y Filosofía. La cultura del cuidado del patrimonio histórico y la salud. Argentina: UNPAedita.

FOUCAULT, M. (2015). La inquietud por la verdad. Argentina: Siglo XXI Editores. Colección Historia del Pensamiento Contemporáneo.

FOUCAULT, M. (2014) Qué es usted Profesor Foucault? Sobre la arqueología y su método. Argentina: Siglo XXI editores. Colección Historia del Pensamiento Contemporáneo.

FOUCAULT, M. (1996) Hermenéutica del sujeto. Argentina: Altamira.

GADAMER, H-G. (2004). Truth and method. Londres: Continuum London. pp 9 a 17

GÓMEZ, O., CARRILLO, G., CÁRDENAS, D. (2017). “Estrategias para el aprendizaje significativo del cuidado humano en Enfermería" disponible en www.researchgate.net

KEROUAC, S. et al (1996) El pensamiento enfermero. España: Masson

MICHALEWICZ, A., PIERRI, C., ARDILLA-GÓMEZ, S. (2014). "Del proceso salud/ enfermedad/atención al proceso salud/enfermedad/cuidado: elementos para su conceptualización" en: Anuario de Investigaciones, Universidad de Buenos Aires, vol XXI: 217-224 disponible en www.redalyc.org

MEDINA MOYA, J., do PRADO, L. (2015). "El curriculum de Enfermería como prototipo de tejné: racionalidad instrumental y tecnológica" en: Texto y Contexto Enfermagem, oct-dic, 18 (4): 617-26 disponible en www.researchgate.net

ROPER, N., LOGAN, W., TIERNEY, A. (1993). Modelo de Enfermería. 3ra edición. México: Interamericana - McGraw - Hill.

RORTY, R. (1979). La filosofía y el espejo de la naturaleza. Madrid: Editorial Cátedra. 
SMITH, M., LIEHR, P. (2018). Middle Range Theory for Nursing, 4ta edición. Nueva York: Springer Publishing Company. Las autoras citan a Conway, 1985

STONEHOUSE, D. (2017). "Roper, Logan \& Tierney's Model of living and Model of nursing. A Support Workers Guide" disponible en http://usir.salford.ac.uk

* Rosana Elisabet Firpo: Licenciada en Enfermería (Universidad de Buenos Aires). Técnica Universitaria en Comunicación Social (Unidad Académica Río Gallegos - Universidad Nacional de la Patagonia Austral). Docente-investigadora. Co-autora de: Pensar el cuidado (2008), Adolescentes y Autocuidado (2011) -el primer libro basado en un proyecto de Extensión de la UARG-UNPA-, El arte de cuidar (2012) y La cultura del cuidado del patrimonio histórico y la salud (2016). Autora del proyecto de investigación "La utilización de simuladores en la enseñanza en Enfermería: salir de la racionalidad instrumental a través de nuevas prácticas pedagógicas" (2018) desde el que se orientó el enfoque pedagógico de David Perkins. En Instagram: @cuidar.comunicación. [E-mail: rosanaelisabet@gmail.com] 